\title{
Plasma lipoprotein(a) levels in patients with slow coronary flow
}

\author{
Habil Yucel ${ }^{1}$, Abdullah Dogan², Ahmet Altinbas ${ }^{2}$, Salaheddin Akcay³, Atilla Icli ${ }^{4}$, Betul Mermi Ceyhan \\ ${ }^{1}$ Department of Cardiology, State Hospital, Isparta, Turkey \\ ${ }^{2}$ Department of Cardiology, Suleyman Demirel University, Medical School, Isparta, Turkey \\ ${ }^{3}$ Department of Cardiology, Celal Bayar University, Medical School, Manisa, Turkey \\ ${ }^{4}$ Department of Cardiology, Ahi Evran University Training and Research Hospital, Kırsehir, Turkey \\ ${ }^{5}$ Department of Biochemistry, Suleyman Demirel University, Medical School, Isparta, Turkey
}

Postep Kardiol Inter 2013; 9, 4 (34): 323-327

DOI: $10.5114 /$ pwki.2013.38404

\section{A bstract}

Introduction: Slow coronary flow (SCF) is a microvascular disorder characterized by delayed opacification of coronary vessels with normal coronary angiogram. It may be due to endothelial dysfunction and diffuse atherosclerosis. Lipoprotein(a) [Lp(a)] is related to cardiovascular events. Plasma Lp(a) levels have not been studied previously in SCF patients.

Aim: We investigated plasma $\operatorname{Lp}(\mathrm{a})$ and fibrinogen levels, and their relation to coronary flow rate in patients with SCF.

Material and methods: This cross-sectional study included 50 patients with SCF and 30 age- and sex-matched controls who had normal coronary arteries and normal flow. Coronary flow rates of patients and controls were counted with the thrombolysis in myocardial infarction (TIMI) frame count. Plasma $L p(a)$ and fibrinogen levels were measured in SCF patients and controls, with routine biochemical tests.

Results: There were no significant differences between the two groups with respect to plasma Lp(a) (21 mg/dl vs. $14 \mathrm{mg} / \mathrm{dl}, p=$ $0.11)$ and fibrinogen $(278 \mathrm{mg} / \mathrm{dl}$ vs. $291 \mathrm{mg} / \mathrm{dl}, p=0.48)$ levels. The TIMI frame count was not correlated with plasma $\operatorname{Lp}(\mathrm{a})(r=0.13$, $p=0.25)$ or fibrinogen $(r=-0.14, p=0.28)$ levels.

Conclusions: Our results show that there is no significant association between SCF and Lp(a) and fibrinogen levels.

Key words: lipoprotein(a), fibrinogen, slow coronary flow, cardiovascular disease.

\section{Introduction}

Slow coronary flow (SCF) is defined as late opacification in the epicardial coronary arteries without significant stenosis based on the coronary images [1]. Although the pathophysiological mechanisms of SCF remain uncertain, there are several hypotheses suggested [2]. Endothelial dysfunction and diffuse atherosclerosis may lead to SCF. Based on this hypothesis, SCF may be a form of early phase of atherosclerosis [3].

Lipoprotein(a) $[\mathrm{Lp}(\mathrm{a})]$ is a cholesterol ester-rich lipoprotein composed of a low-density lipoprotein (LDL) particle and a large glycoprotein, apolipoprotein(a). There is a debate on the association of $L p(a)$ with coronary artery disease (CAD). Some studies have reported a significant association [4-8], but others have not $[9,10]$. Also, plasma fibrinogen levels have been shown to be correlated with $L p(a)$ in patients with and without CAD [11]

\section{Aim}

To our knowledge, there is no study about plasma $\mathrm{Lp}(\mathrm{a})$ levels in patients with SCF. Therefore, we aimed to evaluate plasma Lp(a) levels in patients with SCF and factors associated with the thrombolysis in myocardial infarction (TIMI) frame count.

\section{Material and methods}

One hundred and nineteen consecutive patients with SCF were evaluated. Of all of them, 69 patients were excluded due to hypertension ( $n=25)$, stenotic lesion $\geq 30 \%$ $(n=20)$, diabetes mellitus $(n=13)$, coronary artery ectasia $(n=9)$, hypothyroidism $(n=1)$ and systemic disease $(n=1)$. The remaining 50 patients created the SCF group. The age- and gender-matched control group was composed of 30 patients with normal coronary arteries and normal coronary flow. The indication for coronary

Corresponding author:

Habil Yucel MD, Department of Cardiology, Isparta State Hospital, Gazi Kemal Mah, 1311 Sokak, Tarcan Apt. No: 15/3, Isparta, Turkey, tel.: +90 5325491360, e-mail: habilycl@hotmail.com

Received: 9.11.2012, accepted: 12.08.2013. 
angiography was either the presence of typical angina or positive or equivocal results of noninvasive screening tests for myocardial ischemia in both groups.

Each patient was questioned about major cardiovascular risk factors including family history of CAD, current smoking status, diabetes mellitus, hypertension, hyperlipidemia and obesity. Family history of CAD was defined as CAD in first-grade male relatives before 55 years and in female relatives before 65 years of age. Hypertension was defined as systolic pressure $>140 \mathrm{~mm} \mathrm{Hg}$ and/or diastolic pressure $>90 \mathrm{~mm} \mathrm{Hg}$ or being on antihypertensive medications. Hypercholesterolemia was defined as fasting total cholesterol level $>200 \mathrm{mg} / \mathrm{dl}$ or being on lipid-lowering agents. Diabetes mellitus was defined as a fasting blood glucose level $>126 \mathrm{mg} /$ dl or current use of an anti-diabetic agent. Obesity was defined as body mass index $>30 \mathrm{~kg} / \mathrm{m}^{2}$. Patients who were smoking before hospitalization were considered as smokers.

Non-inclusion criteria were prior myocardial infarction, acute coronary syndromes, moderate to severe valvular heart disease, New York Heart Association (NYHA) class II-IV chronic heart failure, acute heart failure, hypertension, diabetes mellitus, coronary ectasia, peripheral vascular disease, connective tissue disease, renal or hepatic dysfunction, hematological disorders, females taking oral contraceptive pills or hormone replacement therapy, familial hypercholesterolemia, hypothyroidism, including subclinical hypothyroidism, pregnancy, chronic obstructive pulmonary disease or cor pulmonale, history of malignancy, acute or chronic infection, stroke, taking cholesterol-lowering treatment within 4 weeks, use of any drug affecting $\mathrm{L} p(\mathrm{a})$ levels including oestrogens, androgens, anabolic steroids and $\omega-3$ polyunsaturated fatty acids, and regular alcohol use or alcohol use within $48 \mathrm{~h}$. Patients with SCF who had $\geq 30 \%$ diameter stenosis of major coronary arteries were also excluded from the study. The study was approved by the institutional review board, and informed consent was obtained from all the patients.

\section{Coronary angiography}

Selective coronary angiography was performed via a transfemoral approach with the Judkins technique in multiple projections without the use of nitroglycerin. We used iopamidol (lopamiro) as a contrast agent. Coronary arteries were demonstrated with at least four views of the left coronary system using $6 \mathrm{Fr}$ left coronary catheters and two views of the right coronary artery using $6 \mathrm{Fr}$ right coronary catheters at the rate of $15 \mathrm{fps}$ in the same cardiac catheterization laboratory. Coronary blood flow was measured quantitatively using the TIMI frame count. Initial frame count is defined as the frame in which concentrated dye occupies the full width of the proximal coronary artery lumen, touching both borders of the lumen, and forward motion down the artery. The final frame is designated when the leading edge of the contrast column initially arrives at the distal end. Distal end was defined as the distal bifurcation for the left anterior descending artery (LAD), the distal bifurcation of the segment with the longest total distance for the circumflex artery $(C x)$, and the first branch of the posterolateral artery for the right coronary artery (RCA). The LAD coronary artery is usually longer than the other major coronary arteries; the TIMI frame count for this vessel is often higher. To obtain the corrected TIMI frame count for the LAD coronary artery, the TIMI frame count was divided by 1.7 [12]. The mean TIMI frame count for each patient and control subject was calculated by adding the TIMI frame count for $L A D, C x$ and RCA and then dividing the obtained value by three. Due to different durations required for normal visualization of coronary arteries, the corrected cutoff values were $36.28 \pm 2.6$ frames for LAD, $22.28 \pm 4.1$ frames for $C x$, and $20.48 \pm 3$ frames for RCA, as has been reported earlier in the literature [12]. All participants with a TIMI frame count greater than two standard deviations of the previously published range for a particular vessel were considered to have SCF. Any values obtained above these thresholds in 1 of 3 coronary arteries (not all 3) were considered to be SCF in our study. Coronary angiograms and TIMI frame counts were analyzed by two experienced interventional cardiologists blinded to the clinical status and laboratory measurements of the subjects.

\section{Measurements of plasma lipoprotein(a)}

Blood samples were taken in the morning of the examination after overnight fasting and drawn into heparinized tubes for biochemical analysis. For the measurement of $\mathrm{Lp}(\mathrm{a})$, plasma samples were stored frozen at $\leq-30^{\circ} \mathrm{C}$ after immediate centrifugation $(4000 \times \mathrm{g}$ for $10 \mathrm{~min}$ at $4^{\circ} \mathrm{C}$ ). Whole blood count and routine biochemical tests were performed with an autoanalyzer (Aeroset, Abbott, Abbott Park, IL, USA).

Plasma Lp(a) levels were measured as batch with the microenzyme-linked immunosorbent assay (ELISA) method using a total human Lp(a) ELISA assay kit (Immunospec corporation, California, US). All plasma samples for Lp(a) were measured by the same assay and single assay. Results were expressed as $\mathrm{mg} / \mathrm{dl}$.

\section{Statistical analysis}

Data were analyzed with the SPSS software version 11.0 for Windows. Continuous variables were reported as mean \pm standard deviation and categorical variables as percentages. To compare continuous variables, Student $t$ test or Mann-Whitney $U$ test was used where appropriate. Categorical variables were compared with $\chi^{2}$ test. Mann-Whitney $U$ test was used for comparing plasma Lp(a) and fibrinogen levels. Spearman correlation analysis was performed for the analysis of factors correlated 
with mean TIMI frame count. Statistical significance was defined as $p<0.05$.

\section{Results}

Demographic and clinical characteristics of the SCF and control group are presented in Table 1. There were no statistically significant differences between the two groups with respect to body mass index, systolic and diastolic blood pressures, heart rate and risk factors for CAD such as hyperlipidemia, cigarette smoking, family history and obesity (all $p>0.05$ ). The use of aspirin was significantly higher in the SCF group than the control group ( $50 \%$ vs. $17 \%, p=0.004$ ) but there were no significant differences between the two groups with respect to the use of other medications (all $p>0.05$ ).

Laboratory and angiographic characteristics of SCF patients and controls are presented in Table 2 . The groups were similar in terms of creatinine, total cholesterol, triglyceride, low-density lipoprotein (LDL) cholesterol, hemoglobin, hematocrit, white blood cell count and platelet count (all $p>0.05$ ). Fasting glucose was significantly higher in SCF than controls $(p=0.01)$. Inversely, high-density lipoprotein (HDL) cholesterol was significantly lower in SCF $(p=0.03)$.

There were no statistically significant differences between the two groups with respect to plasma Lp(a)

Table 1. Demographic and clinical characteristics of patients with SCF and control group

\begin{tabular}{|c|c|c|c|}
\hline Variable & $\begin{array}{l}\text { SCF group } \\
(n=50)\end{array}$ & $\begin{array}{l}\text { Control group } \\
\quad(n=30)\end{array}$ & Value of $p$ \\
\hline Age [years] & $53.0 \pm 9.1$ & $51.1 \pm 8.1$ & 0.36 \\
\hline Gender (male/female) & $27 / 23$ & $15 / 15$ & 0.73 \\
\hline Hyperlipidemia & $14(28 \%)$ & $4(13 \%)$ & 0.13 \\
\hline Smoking & $10(20 \%)$ & $6(20 \%)$ & 0.99 \\
\hline Family history of CAD & $10(20 \%)$ & $9(30 \%)$ & 0.31 \\
\hline Obesity & $21(42 \%)$ & $8(27 \%)$ & 0.17 \\
\hline Body mass index $\left[\mathrm{kg} / \mathrm{m}^{2}\right]$ & $29.4 \pm 4.1$ & $27.7 \pm 4.0$ & 0.12 \\
\hline Systolic blood pressure [mm Hg] & $119.1 \pm 18.5$ & $122.0 \pm 8.7$ & 0.76 \\
\hline Diastolic blood pressure [mm Hg] & $75.2 \pm 9.0$ & $765 \pm 6.8$ & 0.83 \\
\hline Heart rate [beats/min] & $71.0 \pm 7.3$ & $70.6 \pm 6.7$ & 0.94 \\
\hline
\end{tabular}

SCF-slow coronary flow, CAD - coronary artery disease

Table 2. Laboratory and angiographic characteristics of patients with SCF and control group

\begin{tabular}{|c|c|c|c|}
\hline Variable & $\begin{array}{l}\text { SCF group } \\
(n=50)\end{array}$ & $\begin{array}{c}\text { Control group } \\
(n=30)\end{array}$ & Value of $p$ \\
\hline Fasting glucose [mg/dl] & $100.7 \pm 16.7$ & $91.3 \pm 12.5$ & 0.01 \\
\hline Creatinine [mg/dl] & $0.92 \pm 0.15$ & $0.88 \pm 0.15$ & 0.26 \\
\hline Total cholesterol [mg/dl] & $190.3 \pm 33.1$ & $190.6 \pm 45.4$ & 0.85 \\
\hline Triglycerides [mg/dl] & $150.6 \pm 67.3$ & $130.8 \pm 52.5$ & 0.25 \\
\hline LDL cholesterol [mg/dl] & $112.4 \pm 28.2$ & $109.4 \pm 37.9$ & 0.47 \\
\hline HDL cholesterol [mg/dl] & $47.5 \pm 12.5$ & $54.56 \pm 15.1$ & 0.03 \\
\hline Hemoglobin [g/dl] & $14.4 \pm 1.39$ & $14.3 \pm 1.42$ & 0.89 \\
\hline Hematocrit [\%] & $42.0 \pm 3.7$ & $40.8 \pm 4.4$ & 0.10 \\
\hline White blood cells $\left[\times 10^{9} / 1\right]$ & $7.5 \pm 2.1$ & $7.1 \pm 2.2$ & 0.33 \\
\hline Platelet count $\left[\times 10^{9} / 1\right]$ & $257.5 \pm 62.0$ & $253.6 \pm 76.8$ & 0.95 \\
\hline \multicolumn{4}{|l|}{ TIMI frame count } \\
\hline $\mathrm{CLAD}$ & $32.2 \pm 8.7$ & $15.7 \pm 1.8$ & $<0.001$ \\
\hline$C x$ & $27.0 \pm 9.0$ & $18.5 \pm 2.9$ & $<0.001$ \\
\hline RCA & $20.7 \pm 4.6$ & $16.5 \pm 2.6$ & $<0.001$ \\
\hline Mean TFC & $26.8 \pm 5.5$ & $16.9 \pm 2.0$ & $<0.001$ \\
\hline Fibrinogen $[\mathrm{mg} / \mathrm{dl}]^{*}$ & $278(240-319)$ & $291(250-342)$ & 0.48 \\
\hline Lipoprotein(a) [mg/dl] ${ }^{\star}$ & $21(14-32)$ & $14(6-35)$ & 0.11 \\
\hline
\end{tabular}

*Median (interquartile range). Data presented as mean $\pm S D$

SCF - slow coronary flow, LDL cholesterol - low-density lipoprotein cholesterol, HDL cholesterol - high-density lipoprotein cholesterol, TIMI - thrombolysis in myocardial infarction, $c$-corrected TIMI frame count, LAD - left anterior descending artery, CX-circumflex artery, RCA - right coronary artery, TFC - TIMI frame count 
(21 vs. $14 \mathrm{mg} / \mathrm{dl}, p=0.11$ ) or fibrinogen ( $278 \mathrm{vs.} 291 \mathrm{mg} / \mathrm{dl}$, $p=0.48)$ levels. The TIMI frame count was not correlated with plasma $\mathrm{Lp}(\mathrm{a})(r=0.13, p=0.25)$ or fibrinogen $(r=$ $-0.14, p=0.28$ ) levels. Similarly, plasma Lp(a) levels were not correlated with fibrinogen $(r=0.31, p=0.053)$ in the SCF group.

\section{Discussion}

In the present study, we demonstrated that there was no significant association between $\operatorname{Lp}(\mathrm{a})$ and fibrinogen levels, and SCF. To the best of our knowledge, this is first study investigating plasma Lp(a) levels in patients with SCF.

Diffuse atherosclerosis may play an important role in the pathogenesis of SCF [3]. It has been shown that plasma nitric oxide (NO) levels were significantly lower in patients with SCF than in those with normal coronary flow and were inversely correlated with TIMI frame counts [13]. Several studies have shown significant changes in plasma levels of oxidative stress parameters in patients with SCF compared to healthy individuals [14-16]. Lipoprotein(a) can be an independent risk factor for atherogenic disease [4-8] and also inactivate NO through superoxide anion production and interfere with the stimulation of NO synthase on the endothelium [17]. Thus, it is likely that there may be an association between Lp(a) and SCF according to the results of the studies mentioned above.

Lipoprotein(a) can be related to impaired endothelium-dependent dilatation $[18,19]$. Previous studies have reported that subjects with increased Lp(a) levels $(250 \mathrm{mg} / \mathrm{l}$ and $300 \mathrm{mg} / \mathrm{l}$ ) had reduced acetylcholine-induced coronary vasodilation $[19,20]$ and that increased $L p(a)$ levels may be associated with coronary endothelial dysfunction. Similarly, it has been shown that healthy young subjects with increased $L p(a)$ levels had reduced myocardial vasoreactivity [21].

Several studies have shown that there is an association between plasma Lp(a) levels and CAD [4-8]. Moreover, it is likely that there can be a causal association between elevated $L p(a)$ levels and increased risk of myocardial infarction [8]. A recent large-scale study demonstrated that $L p(a)$ levels were positively associated with cardiovascular events [22]. Similarly, a meta-analysis including 67 prospective studies showed a clear association between elevated $L p(a)$ levels and increased risk of CAD [23].

In the present study, coronary blood flow was measured quantitatively using the TIMI frame count, and potential confounding factors that affect $L p(a)$ such as hypertension [24-26] and diabetes mellitus [25, 27] were eliminated. We examined the association between $\mathrm{Lp}(\mathrm{a})$ and coronary blood flow when atherosclerotic lesions were not recognizable by angiography and there were no significant results. Thus, the present study is the first one investigating the association between $L p(a)$ and coronary blood flow.
Despite studies supporting $\operatorname{Lp}(\mathrm{a})$ concentration as a CAD risk factor, the actual mechanisms linking Lp(a) to atherogenesis remain undefined. Two studies have shown that there was no association between $\operatorname{Lp}(\mathrm{a})$ and atherosclerosis $[9,10]$. Also, when SCF is accepted as a form of early phase of atherosclerosis, our results indicate that there was no association between $L p(a)$ and atherosclerosis, consistent with the findings of previous studies. In a previous study, it was found that Lp(a) levels were not associated with risk for CAD in Turkish people [28]. Similarly, a recent study showed that $L p(a)$ levels were not associated with change in carotid intima-media thickness or brachial flow-mediated dilation [10].

A correlation of circulating fibrinogen with $L p(a)$ levels was found in patients with primary dyslipidemia [29]. Previous clinical studies showed that patients with elevated serum $\operatorname{Lp}(\mathrm{a})$ levels had a significantly increased cardiovascular disease risk together with high fibrinogen levels [30-32]. In this study, there was a weak correlation between $\mathrm{Lp}(\mathrm{a})$ and fibrinogen levels, but plasma fibrinogen levels were comparable in the two groups.

Aspirin can be used in treatment of SCF [33]. It has been demonstrated that mean platelet volume significantly increased in patients with SCF, altered platelet reactivity and aggregation which may require effective anti-platelet therapy [34]. As far as we are aware, there is no study investigating the effects of aspirin in SCF. A recent study showed that aspirin did not improve brachial flow-mediated dilatation (FMD), an index of endothelial function in patients with SCF. However, patients treated with aspirin were free of angina after treatment of SCF [35]. In this study, use of aspirin was significantly higher in the SCF group than the control group. In some previous studies, it was observed that aspirin decreased plasma Lp(a) levels [36-38].

Study limitations - the sample size of this study is small and thus significant findings might not have been obtained. The diagnosis of normal coronary arteries was based on contrast angiograms of the vessel lumen, which underestimate the presence of atherosclerotic plaques. The use of aspirin was significantly higher in the SCF group than the control group.

\section{Conclusions}

This observational study shows that there is no significant association between SCF and Lp(a) and fibrinogen levels. However, further large studies are required to determine the pathophysiological and clinical significance of $L p(a)$ in patients with SCF.

\section{References}

1. Tambe AA, Demany MA, Zimmerman HA, Mascarenhas E. Angina pectoris and slow flow velocity of dye in coronary arteries - a new angiographic finding. Am Heart J 1972; 84: 66-71.

2. Li JJ, Xu B, Li ZC, et al. Is slow coronary flow associated with inflammation? Med Hypotheses 2006; 66: 504-508. 
3. Mangieri E, Macchiarelli G, Ciavolella M, et al. Slow coronary flow: clinical and histopathological features in patients with otherwise normal epicardial coronary arteries. Cathet Cardiovasc Diagn 1996; 37: 375-381.

4. Kamstrup PR. Lipoprotein(a) and ischemic heart disease a causal association? A review. Atherosclerosis 2010; 211: 15-23.

5. Glader CA, Birgander LS, Stenlund H, Dahlén GH. Is lipoprotein(a) a predictor for survival in patients with established coronary artery disease? Results from a prospective patient cohort study in northern Sweden. J Intern Med 2002; 252: 27-35.

6. Saku K, Zhang B, Liu R, et al. Associations among serum lipoprotein(a) levels, apolipoprotein(a) phenotypes, and myocardial infarction in patients with extremely low and high levels of serum lipoprotein(a). Jpn Circ J 1999; 63: 659-665.

7. Erqou S, Kaptoge S, Perry PL, et al. Lipoprotein(a) concentration and the risk of coronary heart disease, stroke, and nonvascular mortality. JAMA 2009; 302: 412-423.

8. Kamstrup PR, Tybjaerg-Hansen A, Steffensen R, Nordestgaard BG. Genetically elevated lipoprotein(a) and increased risk of myocardial infarction. JAMA 2009; 301: 2331-2339.

9. Auer J, Rammer M, Berent R, et al. Lack of association between plasma lipoprotein(a) concentrations and the presence or $a b-$ sence of coronary atherosclerosis. Acta Cardiol 2002; 57: 409-414.

10. Kivimäki M, Magnussen CG, Juonala M, et al. Conventional and Mendelian randomization analyses suggest no association between lipoprotein(a) and early atherosclerosis: the Young Finns Study. Int J Epidemiol 2011; 40: 470-478.

11. Tziomalos K, Athyros VG, Wierzbicki AS, Mikhailidis DP. Lipoprotein a: where are we now? Curr Opin Cardiol 2009; 24: 351-357.

12. Gibson CM, Cannon CP, Daley WL, et al. TIMI frame count: a quantitative method of assessing coronary artery flow. Circulation 1996; 93: 879-888.

13. Sezgin N, Barutcu I, Sezgin AT, et al. Plasma nitric oxide level and its role in slow coronary flow phenomenon. Int Heart J 2005; 46 : 373-382.

14. Li JJ, Qin XW, Li ZC, et al. Increased plasma C-reactive protein and interleukin- 6 concentrations in patients with slow coronary flow. Clin Chim Acta 2007; 385: 43-47.

15. Enli Y, Turk M, Akbay R, et al. Oxidative stress parameters in patients with slow coronary flow. Adv Ther 2008; 25: 37-44.

16. Yildiz A, Gur M, Yilmaz R, et al. Association of paraoxonase activity and coronary blood flow. Atherosclerosis 2008; 197: 257-263.

17. Wanner C, Greiber S, Krämer-Guth A, et al. Lipids and progression of renal disease: role of modified low density lipoprotein and lipoprotein(a). Kidney Int Suppl 1997; 63: S102-S106.

18. Sorensen KE, Celermajer DS, Georgakopoulos D, et al. Impairment of endothelium-dependent dilation is an early event in children with familial hypercholesterolemia and is related to the lipoprotein(a) level. J Clin Invest 1994; 93: 50-55.

19. Schachinger V, Halle M, Minners J, et al. Lipoprotein(a) selectively impairs receptor-mediated endothelial vasodilator function of the human coronary circulation. J Am Coll Cardiol 1997; 30: 927-934.

20. Tsurumi Y, Nagashima H, Ichikawa K, et al. Influence of plasma lipoprotein (a) levels on coronary vasomotor response to acetylcholine. J Am Coll Cardiol 1995; 26: 1242-1250.

21. Sundell J, Laine H, Raitakari OT, et al. Increased lipoprotein(a) is associated with reduced myocardial vasoreactivity in young healthy men. Atherosclerosis 2005; 179: 185-191.

22. Virani SS, Brautbar A, Davis BC, et al. Associations between lipoprotein(a) levels and cardiovascular outcomes in black and white subjects: the Atherosclerosis Risk in Communities (ARIC) Study. Circulation 2012; 125: 241-249.

23. Genser B, Dias KC, Siekmeier R, et al. Lipoprotein (a) and risk of cardiovascular disease - a systematic review and meta analysis of prospective studies. Clin Lab 2011; 57: 143-156.

24. Serban C, Nicola T, Mateescu R, et al. Serum lipoprotein (a) levels in patients with arterial hypertension. Rev Med Chir Soc Med Nat lasi 2010; 114: 798-802.

25. Nasri H. Association of serum lipoprotein (a) with hypertension in diabetic patients. Saudi J Kidney Dis Transpl 2008; 19: 420-427.

26. Sechi LA, De Marchi S. Relationship of lipoprotein(a) to variables of coagulation in hypertensive subjects. J Investig Med 2001; 49: $12-20$.

27. Singla S, Kaur K, Kaur G, et al. Lipoprotein (a) in type 2 diabetes mellitus: Relation to LDL:HDL ratio and glycemic control. Int J Diabetes Dev Ctries 2009; 29: 80-84.

28. Onat A, Yazici M, Hergenç G, et al. Lipoprotein (a) in a population-based study: more significant in Turkish women than men? [Turkish]. Anadolu Kardiyol Derg 2005; 5: 271-277.

29. Ganotakis ES, Gazi IF, Papadakis JA, et al. The relationship between circulating fibrinogen and lipoprotein (a) levels in patients with primary dyslipidemia. Clin Appl Thromb Hemost 2007; 13: 35-42.

30. Cantin B, Després JP, Lamarche B, et al. Association of fibrinogen and lipoprotein(a) as a coronary heart disease risk factor in men (The Quebec Cardiovascular Study). Am J Cardiol 2002; 89: 662-666.

31. Shai I, Rimm EB, Hankinson SE, et al. Lipoprotein (a) and coronary heart disease among women: beyond a cholesterol carrier? Eur Heart J 2005; 26: 1633-1639.

32. Hartmann M, von Birgelen C, Mintz GS, et al. Relation between lipoprotein(a) and fibrinogen and serial intravascular ultrasound plaque progression in left main coronary arteries. J Am Coll Cardiol 2006; 48: 446-452.

33. Jiang $\mathrm{Q}, \mathrm{Wu} F, \mathrm{Wu} Z$, Liang $\mathrm{C}$. Aspirin as a promising drug for slow coronary flow. Med Hypotheses 2012; 79: 701-702.

34. Nurkalem Z, Alper AT, Orhan AL, et al. Mean platelet volume in patients with slow coronary flow and its relationship with clinical presentation. Turk Kardiyol Dern Ars 2008; 36: 363-367.

35. Gunes Y, Gumrukcuoglu HA, Akdag S, et al. Vascular endothelial function in patients with coronary slow flow and the effects of nebivolol. Arq Bras Cardiol 2011; 97: 275-280.

36. Akaike M, Azuma H, Kagawa A, et al. Effect of aspirin treatment on serum concentrations of lipoprotein(a) in patients with atherosclerotic diseases. Clin Chem 2002; 48: 1454-1459.

37. Chasman DI, Shiffman D, Zee RY, et al. Polymorphism in the apolipoprotein(a) gene, plasma lipoprotein(a), cardiovascular disease, and low-dose aspirin therapy. Atherosclerosis 2009; 203: 371-376.

38. Lippi G, Targher G. Optimal therapy for reduction of lipoprotein(a). J Clin Pharm Ther 2012; 37: 1-3. 